\title{
Helleborus purpurascens-Amino Acid and Peptide Analysis Linked to the Chemical and Antiproliferative Properties of the Extracted Compounds
}

\author{
Adina-Elena Segneanu ${ }^{1,2, *}$, Ioan Grozescu ${ }^{1,2}$, Florentina Cziple ${ }^{3}$, Daniel Berki ${ }^{2}$, \\ Daniel Damian ${ }^{1,2}$, Cristina Mariana Niculite ${ }^{4,5}$, Alexandru Florea ${ }^{4,5}$ and Mircea Leabu ${ }^{4,5}$ \\ Received: 1 October 2015 ; Accepted: 27 November 2015 ; Published: 11 December 2015 \\ Academic Editor: Isabel C. F. R. Ferreira \\ 1 National Institute for Research and Development in Electrochemistry and Condensed Matter-INCEMC, \\ Timisoara 300224, Romania; ioangrozescu@gmail.com (I.G.); danieldamian83@gmail.com (D.D.) \\ 2 University Politehnica Timisoara, 2 Piata Victoriei, Timisoara 300006, Romania; berki_daniel@yahoo.com \\ 3 University “Eftimie Murgu”, Resita, 1-4 Traian Vuia, Resita 320085, Romania; cflorentina@yahoo.com \\ 4 Department of Morphological Sciences, University of Medicine and Pharmacy "Carol Davila", \\ 8 Eroilor Sanitari Blvd, Sector 5, Bucharest 050474, Romania; maria.niculite@ivb.ro (C.M.N.); \\ alexflorea1992@gmail.com (A.F.); mircea.leabu@ivb.ro (M.L.) \\ 5 "Victor Babes" National Institute of Pathology, 99-101, Splaiul Independentei, Sector 5, \\ Bucharest 050096, Romania \\ * Correspondence: s_adinaelena@yahoo.com; Tel.: +40-721-072-589
}

\begin{abstract}
There is a strong drive worldwide to discover and exploit the therapeutic potential of a large variety of plants. In this work, an alcoholic extract of Helleborus purpurascens (family Ranunculaceae) was investigated for the identification of amino acids and peptides with putative antiproliferative effects. In our work, a separation strategy was developed using solvents of different polarity in order to obtain active compounds. Biochemical components were characterized through spectroscopic (mass spectroscopy) and chromatographic techniques (RP-HPLC and GC-MS). The biological activity of the obtained fractions was investigated in terms of their antiproliferative effects on HeLa cells. Through this study, we report an efficient separation of bioactive compounds (amino acids and peptides) from a plant extract dependent on solvent polarity, affording fractions with unaffected antiproliferative activities. Moreover, the two biologically tested fractions exerted a major antiproliferative effect, thereby suggesting potential anticancer therapeutic activity.
\end{abstract}

Keywords: antiproliferative activiy; chromatographic techniques; hellebore; thionins; mass spectroscopy

\section{Introduction}

Natural products isolated/extracted from medicinal plants have high potential as new bioactive compounds. As a result, molecules with both low molecular weight (e.g., quinones, cerebrosides, isoflavones, catechol, amines, terpenes, steroids, etc.) and high molecular weight (e.g., polysaccharides, glycoproteins, glycopeptides, proteins, etc.) have been investigated as putative pharmaceutical agents [1].

Helleboreus spp. (family Ranunculaceae) are spontaneous perennial flowering herb plants. In the Romanian spontaneous flora, there are two species of hellebore: Helleborus purpurascens and Helleborus odorus, both of them flowering in early spring [2,3]. Helleborus purpurascens is extremely toxic, yet known since long ago for its folkloric therapeutic effects. It has been used in traditional 
medicine since ancient times for the treatment of various diseases: pain, mental and cardiac disorders, etc. References on the therapeutic use of this herb go back to the time of Hippocrates. In the Middle Ages, the physician and alchemist Paracelsus prepared and proposed an extract of Helleborus as an "elixir" to increase life expectancy [4]. The Romanian researcher Boici used a purified hellebore extract for the preparation of a pharmaceutical product named Boicil, which showed therapeutic activity in rheumatic diseases [5]. The plant extract is used as an anti-rheumatic ointment without any previous investigation of the activity of the individual components of hellebore, therefore, the component(s) responsible for the biological activity of Boicil have not been identified yet. However, recent studies on the antitumor, immune and biological activity of Helleborus extracts revealed the multitude of directions in this field of research targeting the elucidation of the biological potential and cellular mechanisms addressed by various components from this plant [6-8]. Some of the main components of Helleborus purpurascens are glycosides, thionins (helleborine, hellebrine), saponides, resins, lactones, protoanemonine, and minerals [6-10].

The significance of studying the structure of natural peptides was illustrated by numerous pharmacological investigations, identifying various essential properties of these types of compounds such as their low molecular mass, their relatively unique and simple structures, low antigenicity, low toxicity, and an highly biological activity because of their remarkable chemical diversity, steric complexity and high biochemical specificity [1,11-13]. Peptide properties depend on the constituent amino acids and the amino acid sequences in the peptide structure. Modern chemistry offers various methods for biochemical and structural analysis of peptides and glycopeptides. Chromatography and mass spectrometry proved to be efficient techniques for the identification of each component from a complex natural matrix [14].

Thionins are relatively small-sized multiple-cysteine peptides with antimicrobial properties due to their high content of cysteine units [15]. The antitumor and immunomodulating activities of hellebore are attributed to the cytotoxicity of this natural peptide [15-19]. Although there are many studies worldwide on hellebore extracts and their biological activities, research in this area continues to be of great interest because the chemical structure and composition of Helleborus purpurascens have not been fully elucidated yet [6,20-23]. The aim of this study was hence to isolate the amino acids and thionins from an alcoholic extract of Helleborus purpurascens, identify them using spectroscopic and chromatographic techniques and to test their biological activity in terms of antiproliferative effects.

\section{Results and Discussion}

A mandatory step toward separation of target compounds in an extract is the development of an appropriate partition scheme sequence for phytochemicals, employing different types of solvents, in order to remove metabolites and unwanted compounds, such as: lipids, phenols, detergents, resins, lactones, protoanemonins. Depending on the polarity of the solvent used, there emerges a wide distribution of phytochemicals from the hellebore extract in the different fractions recovered [24-27].

\subsection{HPLC Analysis}

The presence of amino acids in hellebore extracts was examined using HPLC. The influence of the solvent (polarity) on the amino acid content in the analyzed samples $\left(\mathrm{S}_{1}, \mathrm{~S}_{4}, \mathrm{~S}_{5}\right.$ and $\left.\mathrm{S}_{6}\right)$ was also taken under consideration. Hence, a qualitative reverse phase HPLC analysis (RP-HPLC) for free amino acids was investigated. Under the same chromatographic conditions, a phenylisothiocyanate (PITC) pre-column derivatization method for standard amino acids was developed. The derivatization step, as a widely used procedure for HPLC analysis of amino acids and peptides, is not necessary if chromatography aims only at separating the amino acids. Comparison of the results of the pre-column PITC derivatization methods for HPLC analysis with those of the RP-HPLC analysis of the hellebore extract could identify its amino acid composition. The proposed RP-HPLC method enabled identification of the amino acids existing in the hellebore fractions $S_{1}, S_{4}, S_{5}$ and $\mathrm{S}_{6}$ (see Table 1). 
The developed RP-HPLC method allowing for separation and identification of the amino acids in the hellebore extract $\left(S_{1}, S_{4}, S_{5}\right.$ and $\left.S_{6}\right)$ is based on the retention times of the used standards.

Table 1. RP-HPLC identification of Helleborus purpurascens amino acids.

\begin{tabular}{|c|c|c|c|c|c|}
\hline $\begin{array}{l}\text { Hellebore } \\
\text { Fraction }\end{array}$ & $\begin{array}{l}\text { Amino } \\
\text { Acids }\end{array}$ & $\begin{array}{l}\text { Retention Time } \\
\text { (Tr) (min) }\end{array}$ & $\begin{array}{l}\text { Peak Area } \\
(\mathrm{mAu} \cdot \mathrm{min})\end{array}$ & $\begin{array}{l}\text { Peak High } \\
\quad(\mathrm{mAu})\end{array}$ & $\begin{array}{c}\text { Relative } \\
\text { Area }\end{array}$ \\
\hline \multirow{7}{*}{$S_{1}$ (ethanolic) } & Cys & 6.319 & 19.7050 & 48.247 & 12.79 \\
\hline & His & 7.21 & 0.6849 & 13.520 & 5.00 \\
\hline & Phe & 7.978 & 9.4757 & 15.515 & 6.15 \\
\hline & Thr & 8.923 & 0.9001 & 3.720 & 5.95 \\
\hline & Glu & 9.958 & 6.4976 & 10.478 & 4.22 \\
\hline & Asn & 22.301 & 108.2632 & 130.637 & 88.89 \\
\hline & Ala & 32.290 & 117.4534 & 110.475 & 76.25 \\
\hline \multirow{10}{*}{$\mathrm{S}_{4}\left(\mathrm{CH}_{2} \mathrm{Cl}_{2}\right)$} & Gly & 6.987 & 0.7801 & 3.567 & 2.39 \\
\hline & His & 7.21 & 0.85 & 14.359 & 4.23 \\
\hline & Phe & 7.89 & 7.854 & 21.464 & 6.43 \\
\hline & Glu & 9.958 & 6.891 & 11.872 & 5.27 \\
\hline & Ser & 12.892 & 1.5890 & 9.671 & 13.561 \\
\hline & Asn & 22.301 & 9.376 & 5.701 & 8.429 \\
\hline & Pro & 29.862 & 1.8539 & 2.257 & 23.13 \\
\hline & Ala & 32.290 & 0.9369 & 1.457 & 9.22 \\
\hline & Lys & 35.802 & 3.2473 & 3.401 & 19.28 \\
\hline & Tyr & 39.347 & 0.5518 & 0.743 & 8.37 \\
\hline \multirow{9}{*}{$\mathrm{S}_{5}$ (n-butanol) } & Gly & 6.987 & 8.627 & 8.672 & 10.391 \\
\hline & His & 7.21 & 0.9138 & 12.188 & 14.57 \\
\hline & Phe & 7.978 & 5.624 & 9.297 & 4.61 \\
\hline & Glu & 9.958 & 0.5390 & 0.948 & 7.94 \\
\hline & Arg & 13.680 & 0.5313 & 0.860 & 17.19 \\
\hline & Val & 25.068 & 0.7673 & 0.754 & 14.83 \\
\hline & Ala & 32.523 & 0.3391 & 0.382 & 9.97 \\
\hline & Lys & 35.802 & 3.287 & 11.720 & 8.134 \\
\hline & Tyr & 39.347 & 10.346 & 23.581 & 12.365 \\
\hline \multirow{4}{*}{$\mathrm{S}_{6}$ (aqueous) } & $\mathrm{C}-\mathrm{C}$ & 6.198 & 12.0542 & 59.209 & 6.52 \\
\hline & Phe & 7.978 & 27.659 & 56.375 & 29.991 \\
\hline & Asp & 23.546 & 15.7821 & 121.368 & 8.53 \\
\hline & Ala & 32.523 & 157.0749 & 139.018 & 54.95 \\
\hline
\end{tabular}

\subsection{GC-MS Analysis}

The content of hellebore extracts in amino acids and peptides and its dependence on solvent type were confirmed by GC-MS analysis. The obtained chromatograms are shown in Figure 1. The mass spectra of the components from the GC-MS chromatograms were compared with those from the NIST/NBS (National Institute of Standards and Technology/National Bureau of Standards) spectral database, and the identified amino acids and peptides are presented in Table 2.

The proposed isolation strategy unraveled the influence of solvent polarity on the efficiency of amino acid and peptide separation from the hellebore extract. Thus, the hexane fraction $\left(\mathrm{S}_{2}\right)$ contains the largest number of amino acids, with the fewest compounds separated in the ethanol fraction $\left(S_{1}\right)$. 
Table 2. GC-MS amino acid identification.

\begin{tabular}{|c|c|c|c|}
\hline Hellebore Fraction & Proposed Structure & Abbreviation & SIM (Selected-Ion Monitoring) \\
\hline \multirow{8}{*}{$\mathrm{S}_{1}$ (ethanol) } & cystine & C-C & 41,42 \\
\hline & glutamic acid & Glu & 38,40 \\
\hline & phenylalanine & Phe & 56,57 \\
\hline & hystidine & Hys & 84 \\
\hline & asparagine & Asn & 155,69 \\
\hline & isoleucine & Ile & 170,130 \\
\hline & threonine & Thr & 180 \\
\hline & $\beta$-alanine & $\beta$-Ala & 216,129 \\
\hline \multirow{13}{*}{$\mathrm{S}_{2}$ (hexane) } & glutamic acid & Glu & 38,40 \\
\hline & cystine & $\mathrm{C}-\mathrm{C}$ & 41,42 \\
\hline & ornitine & Orn & $59,60,61$ \\
\hline & isoleucine & Ile & 170,130 \\
\hline & hystidine & Hys & 84 \\
\hline & proline & Pro & 211 \\
\hline & valine & Val & 158,72 \\
\hline & $\beta$-alanine & $\beta$-Ala & 216,129 \\
\hline & threonine & Thr & 180 \\
\hline & homoserine & HSER & $102,128,143$ \\
\hline & asparagine & Asn & 155,69 \\
\hline & $\alpha$-aminopimelic acid & APA & $198,258,286$ \\
\hline & arginino-succinic acid & ARG-SUC & 326 \\
\hline \multirow{16}{*}{$\mathrm{S}_{3}\left(\mathrm{CCl}_{4}\right)$} & asparagine & Asn & 155,69 \\
\hline & alanine & Ala & 130,70 \\
\hline & glutamic acid & Glu & 38,40 \\
\hline & phenylalanine & Phe & 59,60 \\
\hline & homoserine & HSER & $102,128,143$ \\
\hline & threonine & Thr & 180 \\
\hline & lysine & Lys & 170,129 \\
\hline & glicine & Gly & 116,74 \\
\hline & glycyl-glycine (dipeptide) & Gly-Gly & $117,144,201$ \\
\hline & proline-hydroxyproline (dipeptide) & PHP & 156,186 \\
\hline & lysine-alanine (dipeptide) & Lys-Ala & $170,224,153$ \\
\hline & hystidine & Hys & 84 \\
\hline & 2,4-diamino- $n$-butyric acid & DABA & $203,142,245$ \\
\hline & 1-methylhistidine & 1MHIS & 298 \\
\hline & arginino-succinic acid & ARG-SUC & 326 \\
\hline & $\alpha$-aminopimelic acid & APA & $198,258,286$ \\
\hline \multirow{13}{*}{$\mathrm{S}_{4}\left(\mathrm{CH}_{2} \mathrm{Cl}_{2}\right)$} & valine & Val & 158,72 \\
\hline & $\beta$-alanine & $\beta$-Ala & 216,129 \\
\hline & isoleucine & Ile & 170,130 \\
\hline & glutamic acid & Glu & 38,40 \\
\hline & phenylalanine & Phe & 59,60 \\
\hline & serine & Ser & 146,9 \\
\hline & tyrosine & Tyr & 61,63 \\
\hline & proline-hydroxyproline (dipeptide) & PHP & 156,186 \\
\hline & lysine & Lys & 170,129 \\
\hline & glycine & Gly & 116,74 \\
\hline & glutamine & Gln & 83,186 \\
\hline & hystidine & His & 84 \\
\hline & asparagine & Asn & 155,69 \\
\hline \multirow{7}{*}{$\mathrm{S}_{5}(n$-butanol $)$} & glutamic acid & Glu & 38,40 \\
\hline & phenylalanine & Phe & $59,60,76$ \\
\hline & tyrosine & Tyr & $61,63,94$ \\
\hline & hystidine & His & 84,115 \\
\hline & glycine & Gly & 116,74 \\
\hline & lysine & Lys & 170,129 \\
\hline & valine & Val & 158,72 \\
\hline
\end{tabular}



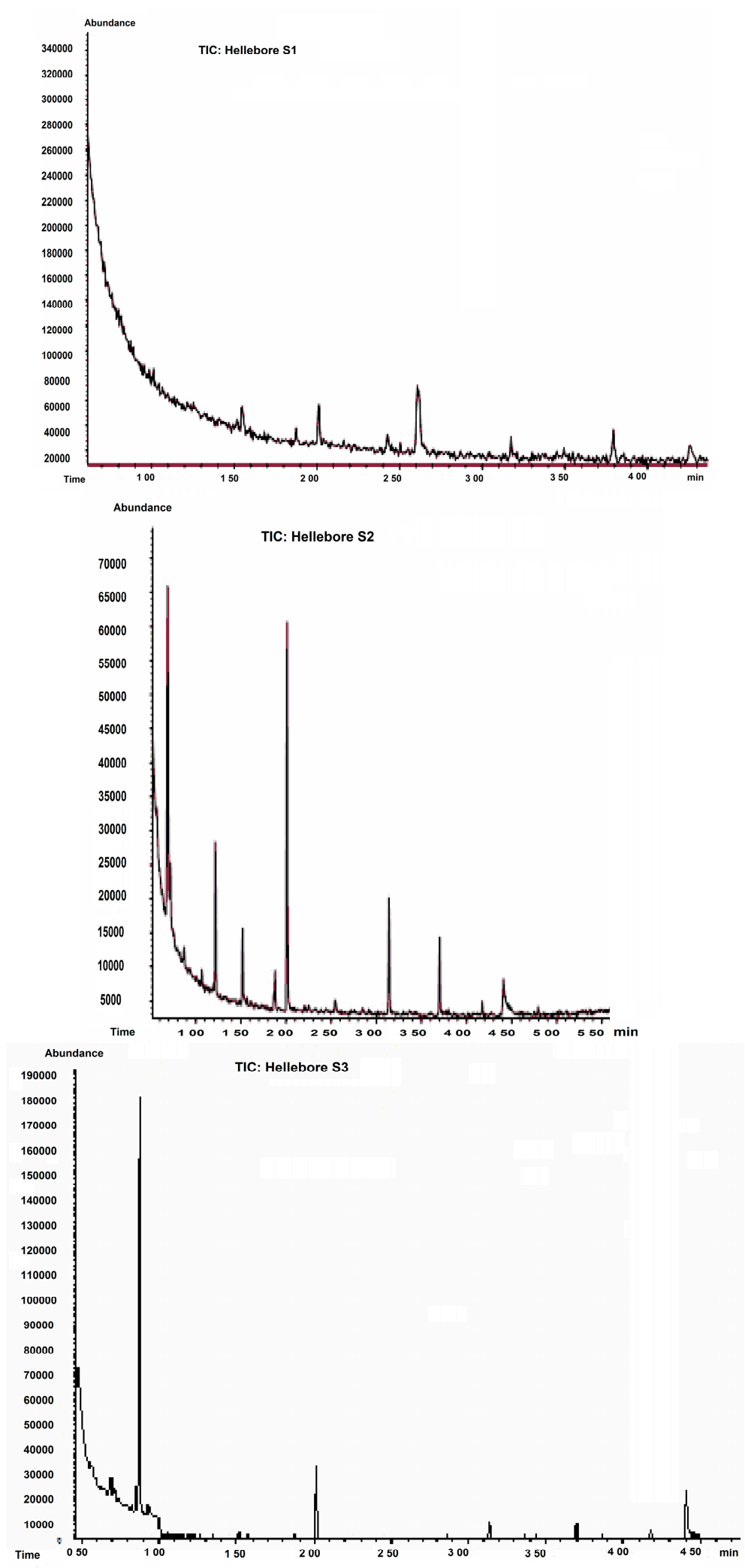

Figure 1. Cont. 

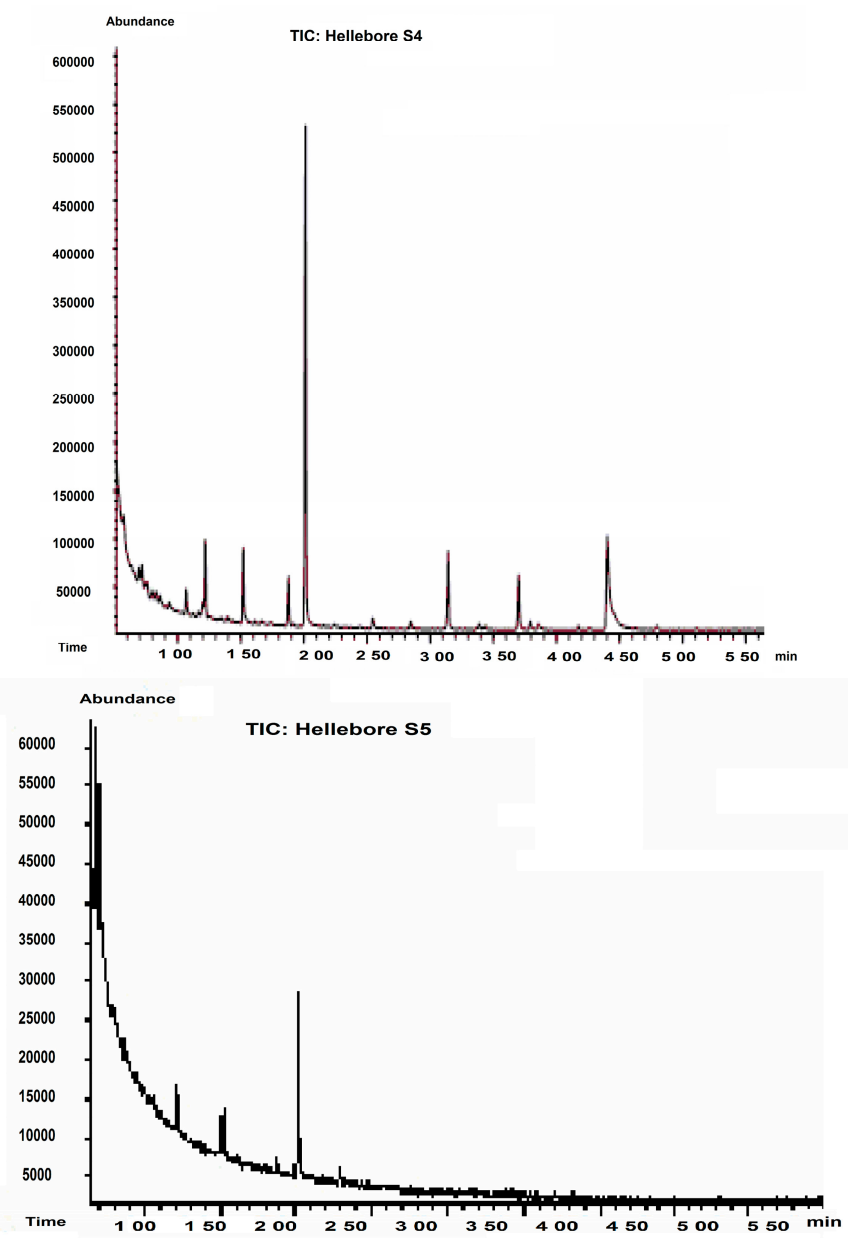

Figure 1. GC-MS chromatogram for $S_{1}-S_{5}$ hellebore extract.

\subsection{Hellebore Thionins Characterized by GC-MS}

The results of the GC-MS for samples $S_{1 T}$ (petroleum ether) and $S_{2 T}$ (acetone) are shown in Figure 2.

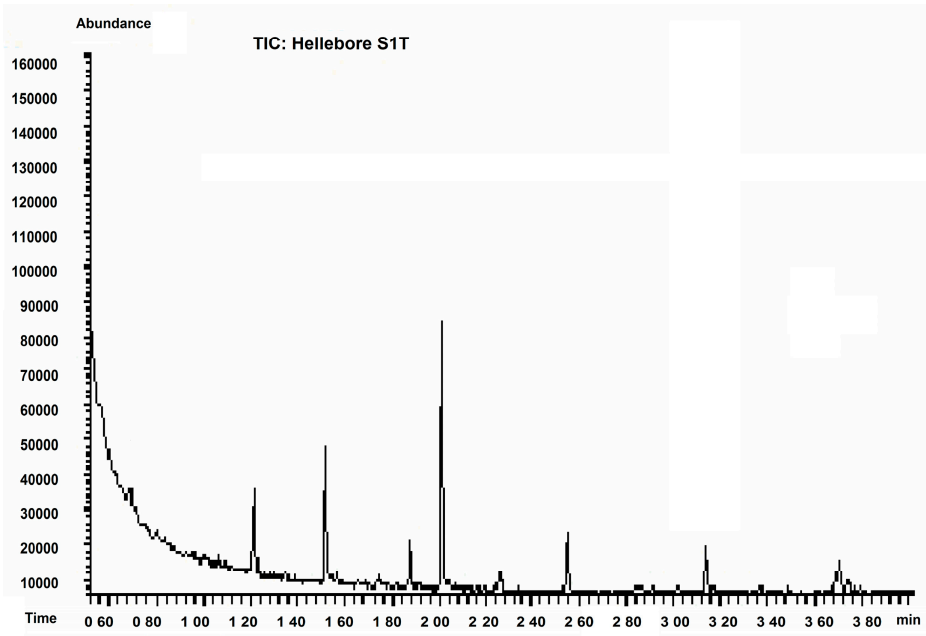

Figure 2. Cont. 


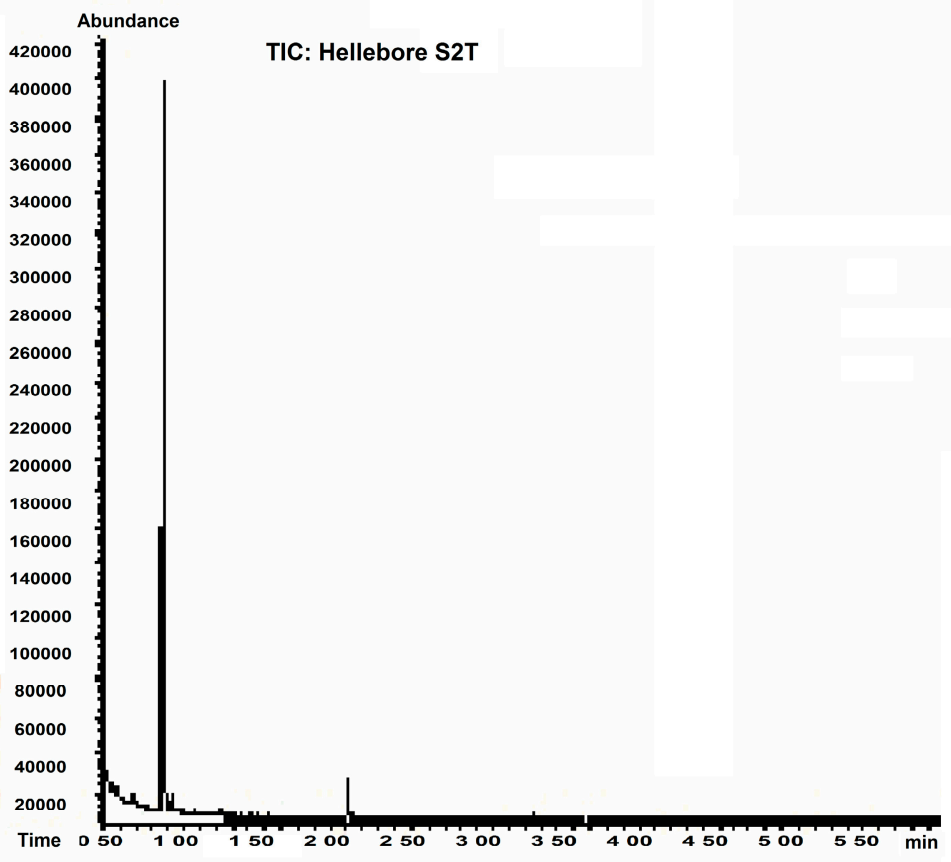

Figure 2. GC-MS chromatogram for $\mathrm{S}_{1 \mathrm{~T}}$ and $\mathrm{S}_{2 \mathrm{~T}}$ hellebore extract.

The compounds identified by mass spectral interpretation through GC-MS analysis are listed in Table 3.

The efficiency of the chosen separation strategy for thionins was evaluated by GC-MS analysis. The results show the influence of solvent polarity on the number of isolated compounds. In the low polarity fraction $\mathrm{S}_{1 \mathrm{~T}}$ (petroleum ether); eleven amino acids and one dipeptide were isolated and identified in the GC-MS chromatogram (Table 3).

Table 3. Thionin GC-MS identification.

\begin{tabular}{cccc}
\hline Hellebore Fraction & Proposed Structure & Abbreviation & SIM \\
\hline & cystine & $\mathrm{C}-\mathrm{C}$ & 41,42 \\
& histidine & His & 84,115 \\
& tyrosine & $\mathrm{Tyr}$ & $61,63,94$ \\
& lysine & $\mathrm{Lys}$ & 170,129 \\
& homoserine & $\mathrm{HSER}$ & $102,128,143$ \\
$\mathrm{~S}_{1 \mathrm{~T}}$ (petroleum ether) & -alanine & $\beta$-Ala & 216,129 \\
& leucine & Leu & 172,86 \\
& phenylalanine & Phe & $59,60,76$ \\
& glutamic acid & Glu & 38,40 \\
& glycine & $\mathrm{Gly}$ & 116,74 \\
& 3-methylcysteine & - & $172,259,130$ \\
& proline-hydroxyproline (dipeptide) & PHP & 156,186 \\
& cystine & $\mathrm{C}-\mathrm{C}$ & 41,42 \\
& glutamic acid & Glu & 38,40 \\
$\mathrm{~S}_{2 \mathrm{~T}}$ (acetone) & tyrosine & Tyr & $61,63,94$ \\
& valine & Val & 158,72 \\
& $\beta$-alanine & $\beta-A l a$ & 216,129 \\
& phenylalanine & Phe & $59,60,76$ \\
\hline
\end{tabular}

\subsection{Nano-ESI-Chip-MS Analysis}

All five hellebore fractions $S_{1}-S_{5}$ were submitted to high-throughput positive nanoESI chip MS and screening under identical solution and instrumental parameters. The obtained mass spectra are 
illustrated in Figures 3-9 while the ion fragments and the corresponding amino acids and peptides (assignments to amino acids and peptide structures) are listed in Table 4.

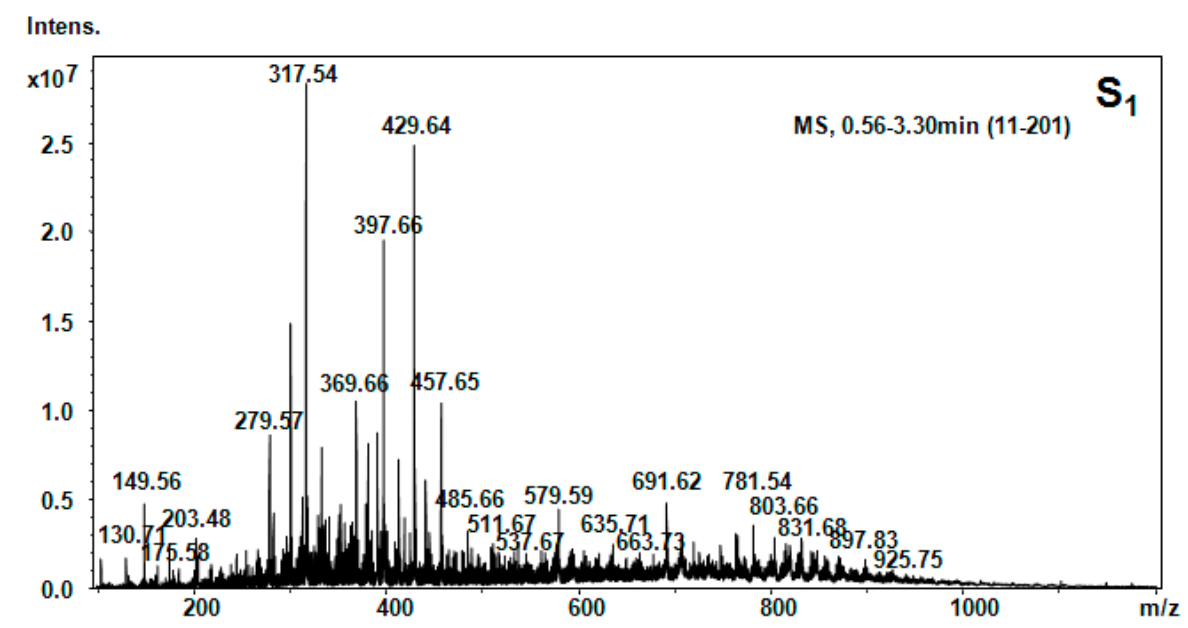

Figure 3. Positive ion mode nano-ESI-Chip-MS forS ${ }_{1}$ hellebore fraction.

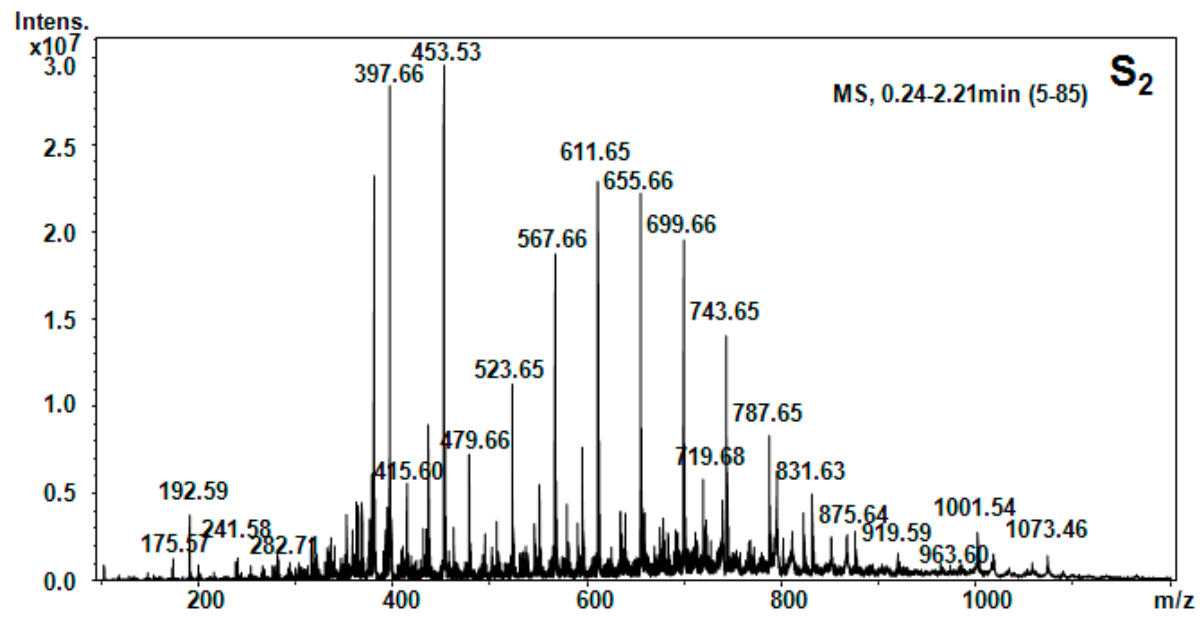

Figure 4. Positive ion mode nano-ESI-Chip-MS for $\mathrm{S}_{2}$ hellebore fraction.

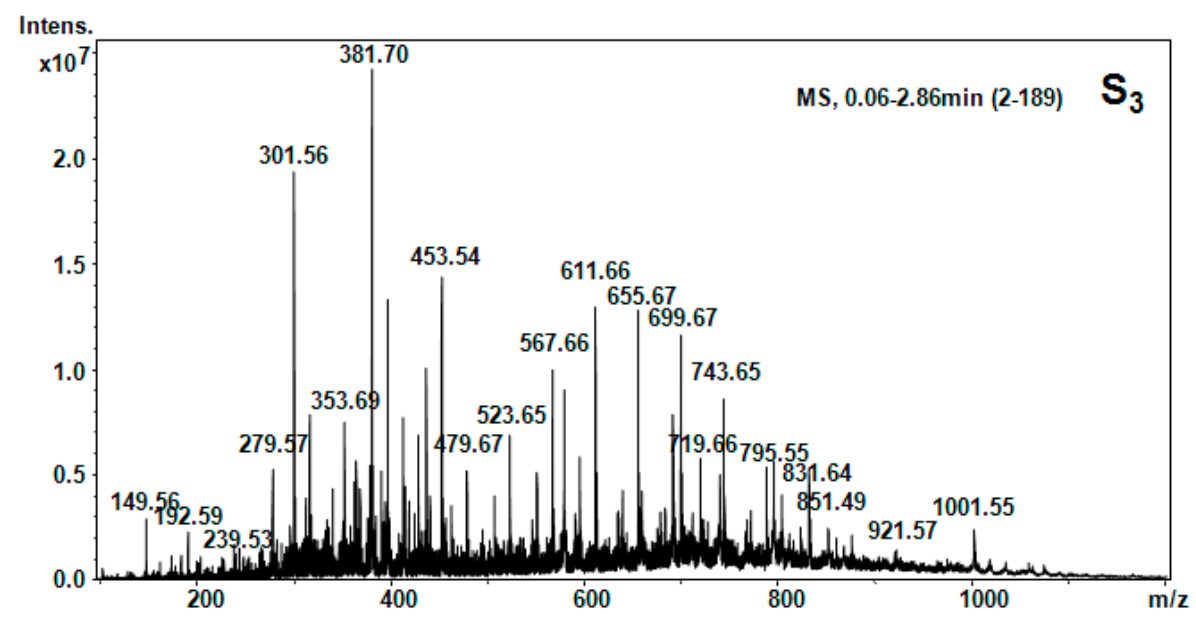

Figure 5. Positive ion mode nano-ESI-Chip-MS for $\mathrm{S}_{3}$ hellebore fraction. 


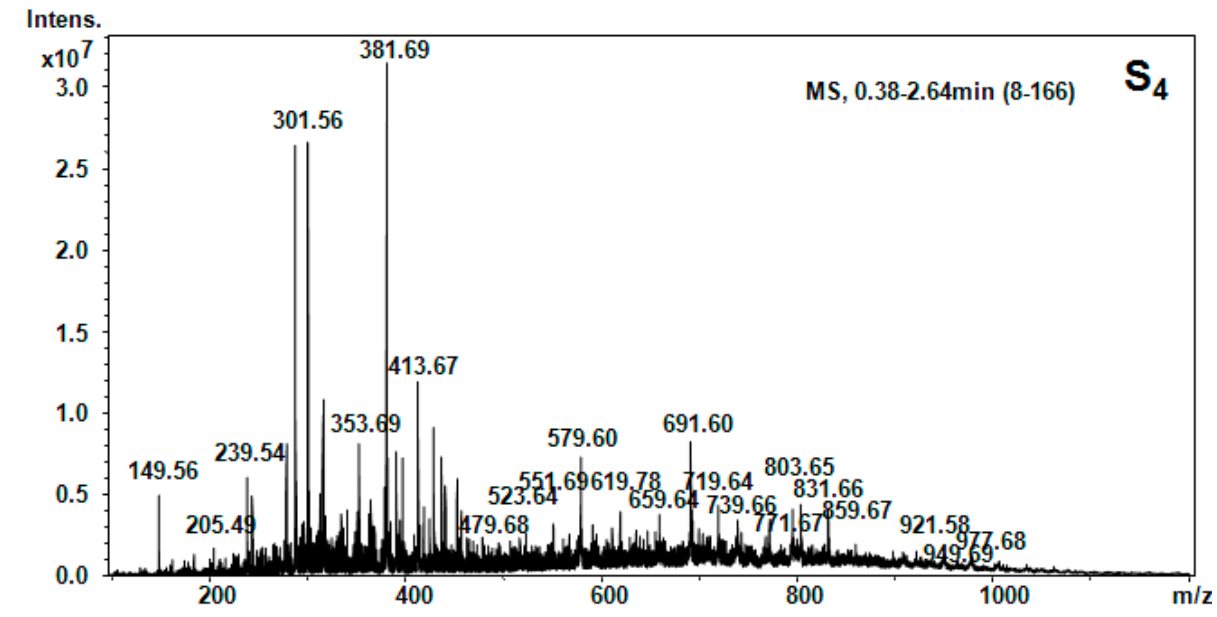

Figure 6. Positive ion mode nano-ESI-Chip-MS for $\mathrm{S}_{4}$ hellebore fraction.

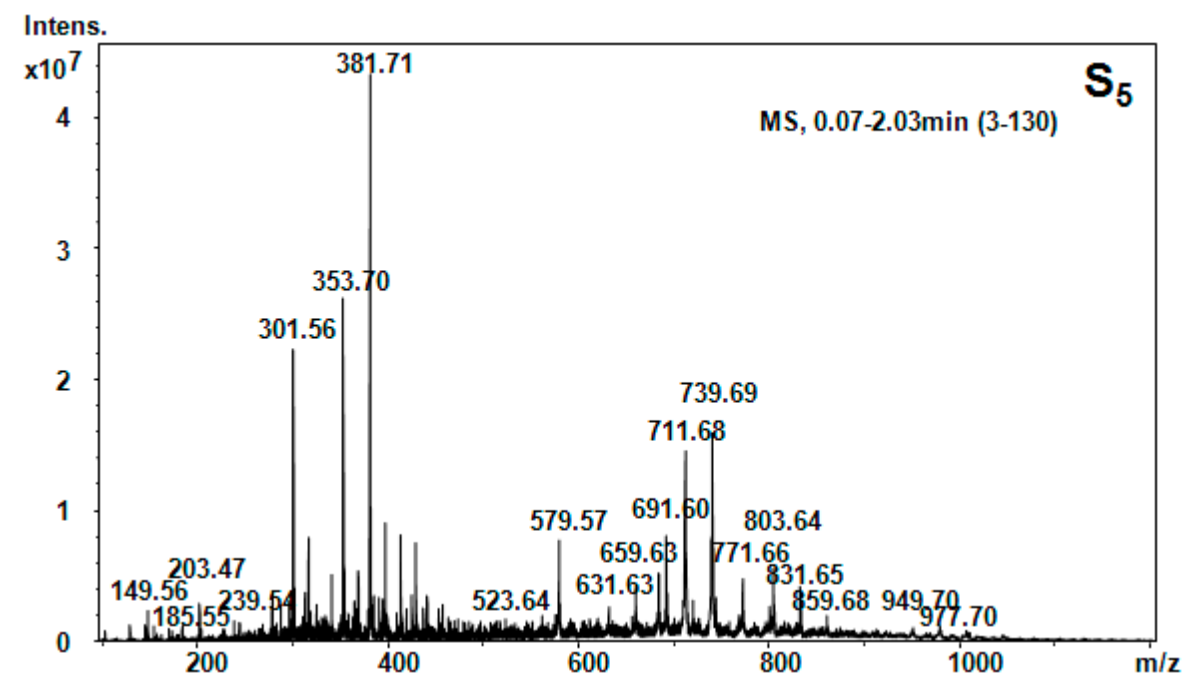

Figure 7. Positive ion mode nano-ESI-Chip-MS for $\mathrm{S}_{5}$ hellebore fraction.

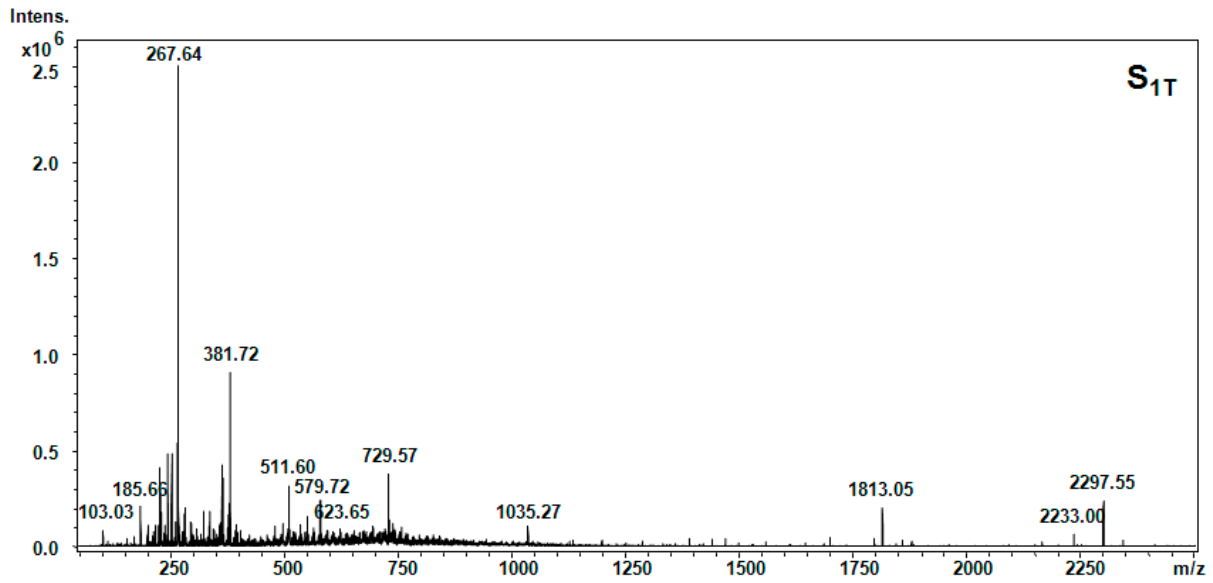

Figure 8. Positive ion mode nano-ESI-Chip-MS of the hellebore thionins in the petroleum ether fraction. 


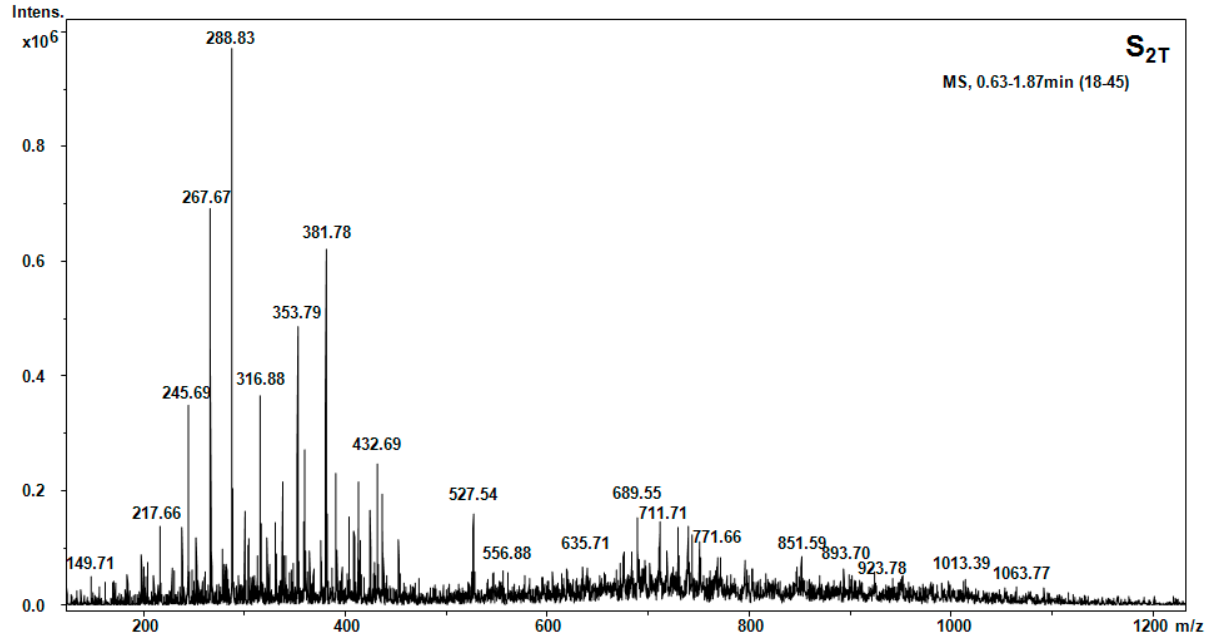

Figure 9. Positive ion mode nano-ESI-Chip-MS of the hellebore thionins in acetone fraction.

Table 4. Amino acids and peptides species in hellebore fractions detected by MS.

\begin{tabular}{|c|c|c|c|c|}
\hline Hellebore Fraction & Type of Molecular Ion & $m / z$ Detected & $m / z$ Calculated & Proposed Structure \\
\hline \multirow{12}{*}{$\mathrm{S}_{1}$ (ethanol) } & {$[\mathrm{M}+2 \mathrm{H}]^{2+}$} & 149.56 & 147.18 & Phe \\
\hline & {$[\mathrm{M}+2 \mathrm{H}]^{2+}$} & 130.71 & 128.14 & Gln \\
\hline & {$[\mathrm{M}+2 \mathrm{H}]^{2+}$} & 175.58 & 173.4 & Arg \\
\hline & {$\left[\mathrm{M}+\mathrm{NH}_{3}\right]^{+}$} & 203.48 & 203.14 & Tryptophan \\
\hline & {$\left[\mathrm{M}+\mathrm{H}_{2} \mathrm{O}+2 \mathrm{H}\right]^{2+}$} & 279.57 & 279.34 & Cys-Arg \\
\hline & {$[\mathrm{M}+2 \mathrm{H}]^{2+}$} & 317.54 & 317.3 & Asp-Glu- $\beta$-Ala \\
\hline & {$[\mathrm{M}+2 \mathrm{H}]^{2+}$} & 369.66 & 369.37 & His-Glu-Thr \\
\hline & {$\left[\mathrm{M}+\mathrm{H}_{2} \mathrm{O}+2 \mathrm{H}\right]^{2+}$} & 397.66 & 397.44 & C-C-His \\
\hline & {$[\mathrm{M}+\mathrm{H}]^{+}$} & 429.64 & 429.46 & Asn- $\beta$-Ala-Ile-Glu \\
\hline & {$[\mathrm{M}+\mathrm{H}]^{+}$} & 457.65 & 457.52 & Phe-His-Thr- $\beta$-Ala \\
\hline & {$\left[\mathrm{M}+\mathrm{H}_{2} \mathrm{O}\right]$} & 579.59 & 579.67 & Thr-Phe-Ile-Glu- $\beta$-Ala \\
\hline & {$\left[\mathrm{M}+\mathrm{H}_{2} \mathrm{O}+\mathrm{H}^{+}\right.$} & 691.62 & 691.8 & Arg-C-C-Phe-Glu \\
\hline \multirow{11}{*}{$\mathrm{S}_{2}$ (hexane) } & {$[\mathrm{M}+2 \mathrm{H}]^{2+}$} & 175.58 & 173.4 & Arg \\
\hline & {$\left[\mathrm{M}+\mathrm{H}_{2} \mathrm{O}+2 \mathrm{H}\right]^{2+}$} & 192.59 & 192.39 & $\beta$-Ala-Thr \\
\hline & {$\left[\mathrm{M}+\mathrm{H}_{2} \mathrm{O}+2 \mathrm{H}\right]^{2+}$} & 397.66 & 397.44 & C-C-His \\
\hline & {$\left[\mathrm{M}+\mathrm{H}_{2} \mathrm{O}+\mathrm{H}\right]^{+}$} & 415.6 & 415.48 & Glu-Pro-Val- $\beta$-Ala \\
\hline & {$\left[\mathrm{M}+\mathrm{H}_{2} \mathrm{O}+\mathrm{H}^{+}\right.$} & 453.53 & 453.5 & Ser-His-Ile-Pro \\
\hline & {$\left[\mathrm{M}+\mathrm{H}_{2} \mathrm{O}+\mathrm{H}\right]^{+}$} & 479.66 & 479.54 & Apa-Ile-Thr- $\beta$-Ala \\
\hline & {$\left[\mathrm{M}+\mathrm{H}_{2} \mathrm{O}+2 \mathrm{H}\right]^{2+}$} & 523.65 & 523.5 & Hser-Orn-His-Asn \\
\hline & {$\left[\mathrm{M}+\mathrm{NH}_{3}\right]^{+}$} & 611.65 & 611.72 & Arg-C-C-Thr-Pro \\
\hline & {$[\mathrm{M}+2 \mathrm{H}]^{2+}$} & 655.66 & 655.6 & Arg-Suc-Asn-HSer-Glu \\
\hline & {$\left[\mathrm{M}+\mathrm{H}_{2} \mathrm{O}+\mathrm{H}\right]^{+}$} & 743.65 & 743.68 & Arg-Succ-Asn-HSer-Glu- $\beta$-Ala \\
\hline & {$\left[\mathrm{M}+\mathrm{H}_{2} \mathrm{O}+\mathrm{H}\right]^{+}$} & 729.57 & 729.39 & Gly-Cystine-Met-Tyr-Hser \\
\hline \multirow{13}{*}{$\mathrm{S}_{3}\left(\mathrm{CCl}_{4}\right)$} & {$[\mathrm{M}+2 \mathrm{H}]^{2+}$} & 149.56 & 147.18 & Phe \\
\hline & {$\left[\mathrm{M}+\mathrm{H}_{2} \mathrm{O}+2 \mathrm{H}\right]^{2+}$} & 192.59 & 192.39 & $\beta$-Ala-Thr \\
\hline & {$\left[\mathrm{M}+\mathrm{H}_{2} \mathrm{O}+2 \mathrm{H}\right]^{2+}$} & 279.57 & 279.34 & Cys-Arg \\
\hline & {$[\mathrm{M}+\mathrm{H}]^{+}$} & 301.56 & 301.56 & 1MHis-Gly-Gly \\
\hline & {$\left[\mathrm{M}+\mathrm{NH}_{3}\right]^{+}$} & 381.70 & 381.32 & Lys-Ala-Phe \\
\hline & {$[\mathrm{M}+\mathrm{H}]^{+}$} & 453.54 & 453.44 & His-Glu-Asn- $\beta$-Ala \\
\hline & {$\left[\mathrm{M}+\mathrm{H}_{2} \mathrm{O}+\mathrm{H}\right]^{+}$} & 479.66 & 479.54 & APA-Ile-Thr- $\beta$-Ala \\
\hline & {$\left[\mathrm{M}+\mathrm{H}_{2} \mathrm{O}+2 \mathrm{H}\right]^{2+}$} & 523.65 & 523.5 & HSer-Orn-His-Asn \\
\hline & {$\left[\mathrm{M}+\mathrm{H}_{2} \mathrm{O}+\mathrm{H}\right]^{+}$} & 552.98 & 552.62 & Pro-Hyp-Phe-Thr-Gly \\
\hline & {$\left[\mathrm{M}+\mathrm{NH}_{3}\right]^{+}$} & 611.65 & 611.72 & Arg-C-C-Thr-Pro \\
\hline & {$[\mathrm{M}+2 \mathrm{H}]^{2+}$} & 655.67 & 655.6 & Arg-Suc-Asn-HSer-Glu \\
\hline & {$\left[\mathrm{M}+\mathrm{H}_{2} \mathrm{O}+\mathrm{H}\right]^{+}$} & 743.65 & 743.68 & Arg-Suc-Asn-HSer-Glu- $\beta$-Ala \\
\hline & {$\left[\mathrm{M}+\mathrm{H}_{2} \mathrm{O}+\mathrm{H}\right]^{+}$} & 729.57 & 729.39 & Gly-Cystine-Met-Tyr-Hser \\
\hline
\end{tabular}


Table 4. Cont.

\begin{tabular}{|c|c|c|c|c|}
\hline Hellebore Fraction & Type of Molecular Ion & $m / z$ Detected & $m / z$ Calculated & Proposed Structure \\
\hline \multirow{15}{*}{$\mathrm{S}_{4}\left(\mathrm{CH}_{2} \mathrm{Cl}_{2}\right)$} & {$[\mathrm{M}+2 \mathrm{H}]^{2+}$} & 149.56 & 147.18 & Phe \\
\hline & {$\left[\mathrm{M}+\mathrm{H}_{2} \mathrm{O}+\mathrm{H}\right]^{+}$} & 205.49 & 205.42 & Val-Ser \\
\hline & {$\left[\mathrm{M}+\mathrm{H}_{2} \mathrm{O}+\mathrm{H}\right]^{+}$} & 239.54 & 239.27 & Gly-Tyr \\
\hline & {$\left[\mathrm{M}+\mathrm{H}_{2} \mathrm{O}+2 \mathrm{H}\right]^{2+}$} & 279.57 & 279.34 & Cys-Arg \\
\hline & {$[\mathrm{M}+\mathrm{H}]^{+}$} & 301.56 & 301.56 & 1MHis-Gly-Gly \\
\hline & {$\left[\mathrm{M}+\mathrm{H}_{2} \mathrm{O}+2 \mathrm{H}\right]^{2+}$} & 353.69 & 353.39 & Gly-Tyr-Ile \\
\hline & {$\left[\mathrm{M}+\mathrm{NH}_{3}\right]^{+}$} & 381.69 & 381.32 & Lys-Ala-Phe \\
\hline & {$\left[\mathrm{M}+\mathrm{NH}_{3}\right]^{+}$} & 413.67 & 413.46 & Hyp-Lys-His \\
\hline & {$\left[\mathrm{M}+\mathrm{H}_{2} \mathrm{O}+\mathrm{H}\right]^{+}$} & 415.6 & 415.48 & Glu-Pro-Val- $\beta$-Ala \\
\hline & {$\left[\mathrm{M}+\mathrm{H}_{2} \mathrm{O}+\mathrm{H}\right]^{+}$} & 479.66 & 479.54 & Apa-Ile-Thr- $\beta$-Ala \\
\hline & {$\left[\mathrm{M}+\mathrm{H}_{2} \mathrm{O}+\mathrm{H}^{+}\right.$} & 552.98 & 552.62 & Pro-Hyp-Phe-Thr-Gly \\
\hline & {$\left[\mathrm{M}+\mathrm{H}_{2} \mathrm{O}+\mathrm{H}\right]^{+}$} & 579.59 & 579.67 & Thr-Phe-Ile-Lys- $\beta$-Ala \\
\hline & {$\left[\mathrm{M}+\mathrm{H}_{2} \mathrm{O}+2 \mathrm{H}\right]^{2+}$} & 619.78 & 619.80 & His-Ser-Val-Tyr-Ile \\
\hline & {$\left[\mathrm{M}+\mathrm{H}_{2} \mathrm{O}+\mathrm{H}\right]^{+}$} & 691.62 & 691.8 & Arg-C-C-Phe-Glu \\
\hline & {$\left[\mathrm{M}+\mathrm{H}_{2} \mathrm{O}+\mathrm{H}\right]^{+}$} & 719.64 & 719.80 & His -Ser-Val-Tyr-Ile-Thr \\
\hline \multirow{9}{*}{$\mathrm{S}_{5}(n$-butanol $)$} & {$[\mathrm{M}+2 \mathrm{H}]^{2+}$} & 149.56 & 147.18 & Phe \\
\hline & {$\left[\mathrm{M}+\mathrm{NH}_{3}\right]^{+}$} & 203.48 & 203.14 & Trn \\
\hline & {$[\mathrm{M}+\mathrm{H}]^{+}$} & 301.56 & 301.56 & 1MHis-Gly-Gly \\
\hline & {$\left[\mathrm{M}+\mathrm{H}_{2} \mathrm{O}+2 \mathrm{H}\right]^{2+}$} & 353.69 & 353.39 & Gly-Tyr-Ile \\
\hline & {$\left[\mathrm{M}+\mathrm{NH}_{3}\right]^{+}$} & 381.69 & 381.32 & Lys-Ala-Phe \\
\hline & {$\left[\mathrm{M}+\mathrm{H}_{2} \mathrm{O}+2 \mathrm{H}\right]^{2+}$} & 523.65 & 523.5 & HSer-Orn-His-Asn \\
\hline & {$\left[\mathrm{M}+\mathrm{H}_{2} \mathrm{O}+\mathrm{H}\right]^{+}$} & 579.59 & 579.67 & Thr-Phe-Ile-Lys- $\beta$-Ala \\
\hline & {$\left[\mathrm{M}+\mathrm{H}_{2} \mathrm{O}+2 \mathrm{H}\right]^{2+}$} & 619.78 & 619.80 & His-Ser-Val-Tyr-Ile \\
\hline & {$\left[\mathrm{M}+\mathrm{H}_{2} \mathrm{O}+\mathrm{H}\right]^{+}$} & 691.62 & 691.8 & Arg-C-C-Phe-Glu \\
\hline \multirow{11}{*}{$\begin{array}{l}\mathrm{S}_{1 \mathrm{~T}} \text { (petroleum } \\
\text { ether) }\end{array}$} & {$[\mathrm{M}+2 \mathrm{H}]^{2+}$} & 103.03 & 103.11 & Thr \\
\hline & {$[\mathrm{M}+\mathrm{H}]^{+}$} & 104.3 & 104.15 & Cys \\
\hline & {$[\mathrm{M}+\mathrm{H}]^{+}$} & 185.66 & 185.25 & $\beta$-Ala-Ile \\
\hline & {$\left[\mathrm{M}+\mathrm{NH}_{3}\right]^{+}$} & 203.48 & 203.14 & Trn \\
\hline & {$\left[\mathrm{M}+\mathrm{NH}_{3}\right]^{+}$} & 267.64 & 267.34 & Phe-Cys \\
\hline & {$\left[\mathrm{M}+\mathrm{NH}_{3}\right]^{+}$} & 381.69 & 381.32 & Lys-Ala-Phe \\
\hline & {$\left[\mathrm{M}+\mathrm{H}_{2} \mathrm{O}+2 \mathrm{H}\right]^{2+}$} & 511.60 & 511.35 & 3-methyl-Cys-Hyp-Pro-Gln \\
\hline & {$\left[\mathrm{M}+\mathrm{H}_{2} \mathrm{O}+\mathrm{H}\right]^{+}$} & 552.98 & 552.62 & Pro-Hyp-Phe-Thr-Gly \\
\hline & {$\left[\mathrm{M}+\mathrm{H}_{2} \mathrm{O}+\mathrm{H}^{+}\right.$} & 579.59 & 579.67 & Thr-Phe-Ile-Glu- $\beta$-Ala \\
\hline & {$\left[\mathrm{M}+\mathrm{H}_{2} \mathrm{O}+\mathrm{H}^{+}\right.$} & 691.62 & 691.8 & Arg-C-C-Phe-Glu \\
\hline & {$\left[\mathrm{M}+\mathrm{H}_{2} \mathrm{O}+\mathrm{H}\right]^{+}$} & 729.57 & 729.39 & Gly-C-C-Met-Tyr-HSer \\
\hline \multirow{17}{*}{$\mathrm{S}_{2 \mathrm{~T}}$ (acetone) } & {$[\mathrm{M}+2 \mathrm{H}]^{2+}$} & 103.03 & 103.11 & Thr \\
\hline & {$[\mathrm{M}+\mathrm{H}]^{+}$} & 104.3 & 104.15 & Cys \\
\hline & {$[\mathrm{M}+\mathrm{H}]^{+}$} & 185.66 & 185.25 & $\beta$-Ala-Ile \\
\hline & {$\left[\mathrm{M}+\mathrm{NH}_{3}\right]^{+}$} & 203.48 & 203.14 & Trn \\
\hline & {$\left[\mathrm{M}+\mathrm{NH}_{3}\right]^{+}$} & 267.64 & 267.34 & Phe-Cys \\
\hline & {$\left[\mathrm{M}+\mathrm{H}_{2} \mathrm{O}+2 \mathrm{H}\right]^{2+}$} & 288.83 & 288.33 & Met-His \\
\hline & {$[\mathrm{M}+\mathrm{H}]^{+}$} & 301.56 & 301.56 & 1MHis-Gly-Gly \\
\hline & {$\left[\mathrm{M}+\mathrm{H}_{2} \mathrm{O}+2 \mathrm{H}\right]^{2+}$} & 353.69 & 353.39 & Gly-Tyr-Ile \\
\hline & {$\left[\mathrm{M}+\mathrm{NH}_{3}\right]^{+}$} & 381.69 & 381.32 & Lys-Ala-Phe \\
\hline & {$\left[\mathrm{M}+\mathrm{H}_{2} \mathrm{O}+2 \mathrm{H}\right]^{2+}$} & 432.69 & 432.46 & Phe-His-Gly- $\beta$-Ala \\
\hline & {$\left[\mathrm{M}+\mathrm{H}_{2} \mathrm{O}+2 \mathrm{H}\right]^{2+}$} & 523.65 & 523.5 & HSer-Orn-His-Asn \\
\hline & {$\left[\mathrm{M}+\mathrm{H}_{2} \mathrm{O}+2 \mathrm{H}\right]^{2+}$} & 527.54 & 527.60 & Orn-Val-Glu-Phe \\
\hline & {$\left[\mathrm{M}+\mathrm{H}_{2} \mathrm{O}+\mathrm{H}\right]^{+}$} & 552.98 & 552.62 & Pro-Hyp-Phe-Thr-Gly \\
\hline & {$\left[\mathrm{M}+\mathrm{H}_{2} \mathrm{O}+\mathrm{H}^{+}\right.$} & 579.59 & 579.67 & Thr-Phe-Ile-Glu- $\beta$-Ala \\
\hline & {$\left[\mathrm{M}+\mathrm{H}_{2} \mathrm{O}+\mathrm{H}\right]^{+}$} & 689.55 & 689.35 & Ile-C-C-Trp-Hyp \\
\hline & {$\left[\mathrm{M}+\mathrm{H}_{2} \mathrm{O}+\mathrm{H}\right]^{+}$} & 691.62 & 691.8 & Arg-C-C-Phe-Glu \\
\hline & {$\left[\mathrm{M}+\mathrm{H}_{2} \mathrm{O}+\mathrm{H}\right]^{+}$} & 729.57 & 729.39 & Gly-C-C-Met-Tyr-HSer \\
\hline
\end{tabular}

To elucidate the chemical composition of the hellebore fractions, we have developed a fully automated new mass spectrometry method based on nanoESI high-capacity ion trap (HCT), in a similar manner as described in the literature [28]. The mass spectrometry results confirmed the chemical structures detected by chromatographic techniques. Although, the developed chromatographic analysis method offered information about the great majority of the chemical species present in the hellebore extracts, mass spectrometry provided many more data on the complexity of the protein structures found in the studied hellebore fractions. Comparison of the screened mass spectra of the hellebore fractions $S_{1}-S_{5}$ provided direct evidence of the variable composition of each fraction due, primarily, to the solvent employed. The investigation of thionin 
composition from the hellebore fractions $\mathrm{S}_{1 \mathrm{~T}}$ and $\mathrm{S}_{2 \mathrm{~T}}$ highlighted the same compounds identified by previous analytical methods. The information gained from this study corroborates with the existing data from the literature $[29,30]$. Our results revealed that the proposed methods proved to be useful tools for the separation and identification of individual compounds from complex natural mixtures.

\subsection{Biological Activity Investigation}

The usefulness of timelapse videomicroscopy in the investigation of cell behavior and biological activity of different pharmaceuticals was previously argued upon and proven [31,32]. Here, studies were conducted to assess, in a cancer cell line (HeLa), the effects of two fractions obtained from the alcoholic extract of Helleborus purpurascens. As shown in Figure 10, the cells treated with various concentrations of $S_{5}$ (Figure 10A) and $S_{2 T}$ (Figure 10D) failed to multiply. The number of mitoses significantly decreased for treated cells for all concentrations used of both $\mathrm{S}_{5}$ (Figure 10B) and $\mathrm{S}_{2 \mathrm{~T}}$ (Figure 10E). The number of dead cells did not spectacularly increase, although it varied significantly in comparison to untreated cells for both $S_{5}$ (Figure 10C) and $S_{2 T}$ (Figure 10F).
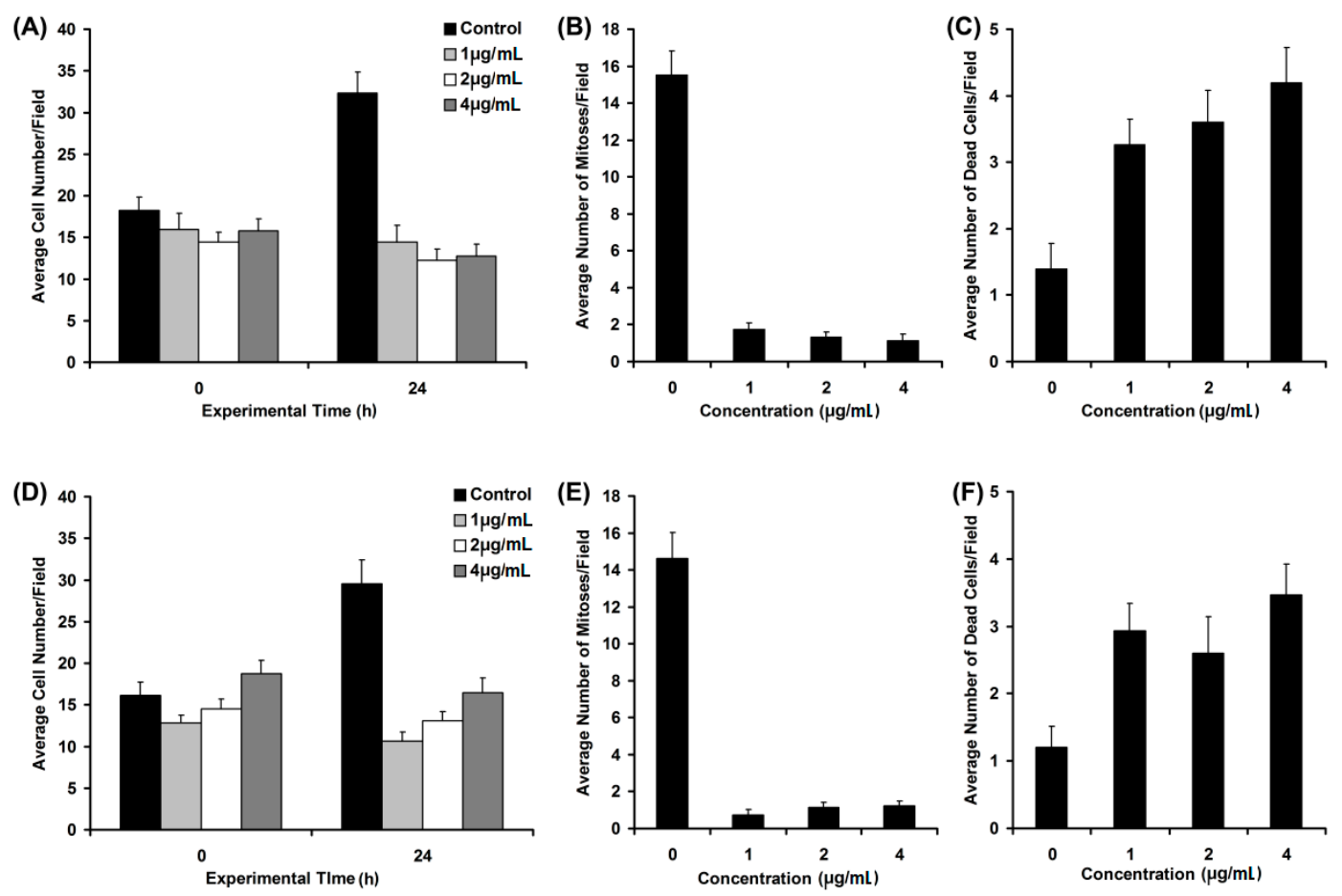

Figure 10. Effects of $S_{5}(\mathbf{A}-\mathbf{C})$ and $S_{2 T}(\mathbf{D}-\mathbf{F})$ fractions on HeLa cell behavior monitored by timelapse videomicroscopy. Untreated cells have grown as usual, doubling their number in $24 \mathrm{~h}$ (A-D, control black bars).

Both tested fractions $\left(\mathrm{S}_{5}\right.$ and $\left.\mathrm{S}_{2 \mathrm{~T}}\right)$ inhibited cell proliferation at significantly low concentrations, as shown by HeLa cell growth in the absence or presence of compounds (Figure 10A,D). Cells that were not treated proliferated and doubled their number after a $24 \mathrm{~h}$ monitoring. The number of observed mitoses was significantly higher in the absence of the tested compounds (Figure 10B,E). It is noteworthy that the number of dead cells during the experimental period of $24 \mathrm{~h}$, although significantly increased after treatment, still remained very low (Figure 10C,F), indicating a reduced cytotoxic effect of both fractions $\mathrm{S}_{5}$ and $\mathrm{S}_{2 \mathrm{~T}}$, isolated from the Helleborus purpurascens alcoholic extract. With regard to the dynamics of the identified effects, fraction $S_{5}$ arrested cell division almost completely after $6 \mathrm{~h}$, when the highest concentration was used. For the medium and low concentration, the same effect was observed at about $12 \mathrm{~h}$ and beyond. A very interesting result was observed in terms of kinetics of the few mitoses still occurring in the treated samples. If for untreated 
HeLa cells a starting mitosis succeeded finalizing its cytokinesis stage in no more than half an hour, treated cells starting mitosis needed a significantly longer period until cytokinesis occurred, meaning more than $3 \mathrm{~h}$ (Figure 11).

Accordingly, an untreated cell, spread on the culture surface (Figure 11A), started a mitotic process (Figure 11B) and accomplished cytokinesis after $20 \mathrm{~min}$ (Figure 11C), whereas the two daughter cells had already spread, $1 \mathrm{~h}$ after mitosis has begun (Figure 11D). A treated cell (Figure 11E) that entered mitosis (Figure 11F) needed $200 \mathrm{~min}$ to accomplish cytokinesis (Figure 11G), and $90 \mathrm{~min}$ more to spread after the mitotic event (Figure 11H), meaning that it needed $4 \mathrm{~h}$ and $50 \mathrm{~min}$ to finalize a significantly, extremely slow division. This observation supports the conclusion that compounds in both $\mathrm{S}_{5}$ and $\mathrm{S}_{2 \mathrm{~T}}$ fractions exerted a major antiproliferative effect, recommending them as potential anticancer therapeutic agents. There are very strong evidences on the fact that in those fractions was not identified other molecules, except amino acids and tionins. However, further investigations are needed to assert if the identified biological activity is due exclusively to amino acids, thionins or to other small molecules, as yet not identified by the analytical techniques used.
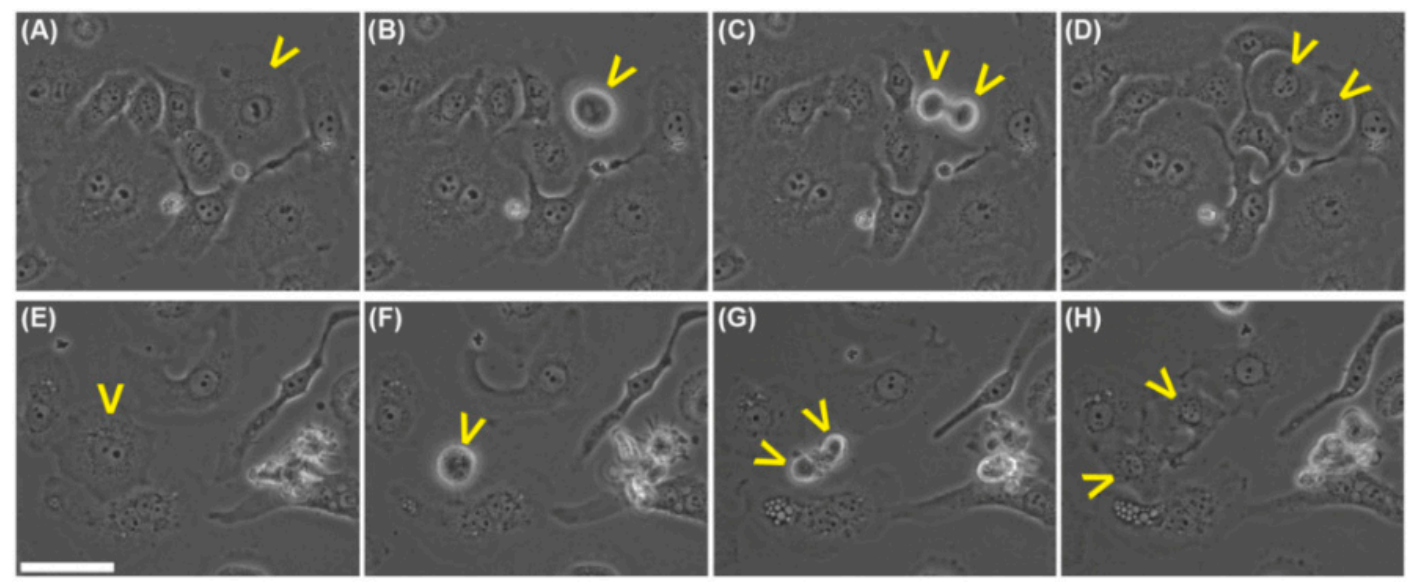

Figure 11. Phase contrast micrographs showing cells before, during and at the end of mitosis. Yellow arrowheads indicate cells of interest. (A) Control cells before starting any mitosis; (B) A control cell is rounding up starting its mitosis; (C) The same cell as in (B) 20 min later, during cytokinesis; (D) The two daughter cells, resulting in mitosis, significantly spread $1 \mathrm{~h}$ after mitosis was started; (E) Treated cells before starting any mitosis; (F) A treated cell rounded up and starting its mitosis; (G) The same cell as in (F), 200 min later, accomplishing its cytokinesis; (H) The daughter cells showing significant spreading $90 \mathrm{~min}$ later, that means almost $5 \mathrm{~h}$ after mitosis has started. White scale bar in (E) represents $50 \mu \mathrm{m}$, and is identical for all the images.

\section{Experimental Section}

\subsection{General Information}

All used reagents were analytical grade. Amino acids were acquired from Applichem (St. Louis, MO, USA) and Alfa Aesar (Ward Hill, MA, USA) and the solvents (methanol, acetone, dichloromethane, carbon tetrachloride, hexane, and $n$-butanol, petroleum ether, ethanol) from VWR (Wien, Austria). Pyridine and phenylisothiocyanate (PITC) were purchased from Alfa Aesar.

Plant roots and rhizomes were collected in February 2011, in Arieseni, Alba (Romania) and identified by Dr. Dana Bobit (vice-president of the Romanian Ethnopharmacology Society, Dacia Plant SRL Brasov, Romania). Voucher samples: 00321 and 00322 were deposited at the Botanical Garden Cluj Napoca, Romania. 


\subsection{Chromatographic Techniques}

The content of amino acids and peptides from the hellebore samples was analyzed by high performance liquid chromatography (HPLC 3000, Ultimate, Dreieich, Germany) using a photodiode array detector and an EZ: faast $4 \mathrm{u}$ AAA-MS Column $(250 \times 3 \mathrm{~mm}$ ID). Qualitative analysis of free amino acids and peptides was performed on a GC-MS 7890A-5975C system (Agilent, Waldbronn, Germany) using the EZ: faast GC-MS free amino acids kit and ZB-AAA GC column (Phenomenex, Torrance, CA, USA). The employed analysis conditions were the standard conditions provided with the kit.

\subsection{Spectroscopy Techniques}

Mass spectrometry was conducted on a High Capacity Ion Trap Ultra mass spectrometer (HCT Ultra, PTM discovery) from Bruker Daltonics (Bremen, Germany). All mass spectra were acquired in the mass range $m / z 100-3000$, with a scan speed of 2.1 scan per second.

\subsection{Isolation Strategy for Amino Acids and Peptides}

The nature of the target bioactive compounds is influenced by the type of solvent employed. In general, a mixture of solvents was preferred to achieve the selective extraction of interested bioactive compounds from natural products. A preliminary sample was prepared using several solvents with different polarities (ethanol, dichloromethane, carbon tetrachloride, hexane, and $n$-butanol) in order to extract the corresponding phytochemicals from the hellebore extract. It was observed that in fractions with low and medium polarity (dichloromethane, carbon tetrachloride and hexane) lipophilic compounds were usually found, including: fatty acids, terpenes, steroids, peptides, depsipeptides, etc. High polarity fractions contained saponins, amino acids, alkaloids, sugars, etc. [24-26,33].

The solvent from a $50 \mathrm{~mL}$ hellebore hydroalcoholic $(1.5: 1 \mathrm{v} / v$; plant extract:alcohol ratio $=1: 10)$ extract was removed under vacuum, the residue was dissolved in water and subsequently extracted with the following solvents: $n$-hexane $\left(S_{2}\right)$, carbon tetrachloride $\left(S_{3}\right)$, dichloromethane $\left(S_{4}\right)$ and $n$-butanol $\left(\mathrm{S}_{5}\right)$. Each of the six organic fractions: $\mathrm{S}_{1}$ (ethanolic), $\mathrm{S}_{2}$ (hexane), $\mathrm{S}_{3}\left(\mathrm{CCl}_{4}\right), \mathrm{S}_{4}\left(\mathrm{CH}_{2} \mathrm{Cl}_{2}\right), \mathrm{S}_{5}$ (n-butanol), and $\mathrm{S}_{6}$ (aqueous) was analyzed by spectroscopic and chromatographic methods.

\subsection{Derivatization of Standard Amino Acids}

A pre-column derivatization method using PITC (phenylisothiocyanate) or Edman's Reagent was carried out. The obtained phenylisothiocyanate derivatives (PITC-amino acids) were analyzed by reverse-phase high-performance liquid-chromatography [34-39]. This method was chosen because the derivatization reagent reacts easily with all amino acids in an alkaline milieu and produces stable products. A sample $(1.5 \mu \mathrm{mol})$ of standard amino acids is mixed with $1 \mathrm{~mL}$ derivatization reagent: pyridine: $\mathrm{H}_{2} \mathrm{O}$ (40:60) and $15 \mathrm{mg}$ PITC. The obtained mixture is heated at $40{ }^{\circ} \mathrm{C}$ for $1 \mathrm{~h}$. Then, $1 \mathrm{~mL}$ $\mathrm{H}_{2} \mathrm{O}$ is added. The excess of PITC is removed by washing four times with $2 \mathrm{~mL}$ of benzene. The aqueous phase is evaporated and dried in vacuum. The residues are dissolved in $1.5 \mathrm{~mL}$ methanol and analyzed on HPLC.

\subsection{Thionins}

For the separation of thionins, the solvent from a $50 \mathrm{~mL}$ hellebore hydroalcoholic extract was removed under vacuum and the resulting residue was extracted successively with petroleum ether $(20 \mathrm{~mL})$ and acetone $(20 \mathrm{~mL})$. Both isolated fractions $-S_{1 \mathrm{~T}}$ (petroleum ether) and $\mathrm{S}_{2 \mathrm{~T}}$ (acetone)-were investigated by analytical methods to reveal the presence of the isolated compounds. 


\subsection{GC-MS Separation Conditions}

The standard analysis conditions were according to instructions from the kit: Oven: $30{ }^{\circ} \mathrm{C}$ (hold $1 \mathrm{~min}$ ) to $40^{\circ} \mathrm{C}$ at $30^{\circ} \mathrm{C} / \mathrm{min}$ (hold $10 \mathrm{~min}$ ) to $360^{\circ} \mathrm{C}$ (hold $1 \mathrm{~min}$ ); Equilibration time: $1 \mathrm{~min}$; Injection: split 1: 15; $250{ }^{\circ} \mathrm{C} ; 2 \mu \mathrm{L}$; Carrier Gas: Helium $1.1 \mathrm{~mL} / \mathrm{min} ; 110{ }^{\circ} \mathrm{C}$; Inlet pressure: $5.824 \mathrm{kPa} / \mathrm{min}$; Detector: MS; Mode: Scan Transfer Line Temperature: $250{ }^{\circ} \mathrm{C}$; Analyzer Type: Electron Energy: $70 \mathrm{eV}$.

\subsection{HPLC-DAD Separation Conditions}

The separation was performed by isocratic elution at Flow Rate: $0.6 \mathrm{~mL} / \mathrm{min} ., \mathrm{Col}$. Temp.: $35^{\circ} \mathrm{C}$ and with UV detection $(\lambda=230 \mathrm{~nm})$. Eluent A: $\mathrm{H}_{2} \mathrm{O}$-acetonitrile-TFA $\left(94.96 \% \mathrm{H}_{2} \mathrm{O}, 5 \%\right.$ acetonitrile, 0.037\% TFA), Eluent B: $\mathrm{H}_{2} \mathrm{O}$-acetonitrile-TFA (94.96\% acetonitrile, $5 \% \mathrm{H}_{2} \mathrm{O}, 0.037 \%$ TFA).

\subsection{Mass Spectrometry Analysis}

Tandem mass spectrometry was carried out by collision-induced dissociation (CID) using He as the collision gas. For MS/MS sequencing, the precursor ions were selected within an isolation width of $2 \mathrm{u}$. Fully automated chip-nano ESI performed in a NanoMate 400 robot incorporating ESI chip technology (Advion BioSciences, Ithaca, NY, USA) coupled on a High Capacity Ion Trap Ultra mass spectrometer (HCT Ultra, PTM discovery, Bruker Daltonics). The robot was controlled and manipulated by the ChipSoft software (Advion BioSciences) operating under Windows. The position of the electrospray chip was adjusted to the sampling cone potential so as to give rise to an optimal transfer of the ionic species into the mass spectrometer. In order to avoid any contamination in all experiments, a glass-coated microtiter plate was used. Five $\mu \mathrm{L}$ aliquots of the working sample solutions were loaded onto the 96-well plate. The robot was programmed to take up the whole volume of sample, followed by $2 \mu \mathrm{L}$ of air into the pipette tip and then deliver the sample on the inlet side of the microchip. Each nozzle has an internal diameter of $2.5 \mu \mathrm{m}$ and under the given condition delivered a flow rate of about $200 \mathrm{~nL} / \mathrm{min}$. NanoESI process was initiated by applying voltages within 1.5 to $1.8 \mathrm{kV}$ and a head pressure of $0.5-0.7$ p.s.i. After spray initialization, infusion parameters were optimized: ESI voltage in pipette tip, voltage and desolvation gas flow. The values of Nano-ESI source parameter, ESI capillary, cone potential and desolvation gas (Nitrogen) were optimized to achieve an efficient ionization and produce the optimum transfer of ions in MS. Measurement parameters were: Capillary voltage $1 \mathrm{kV}$; Counter electrode voltage (cone voltage) $60 \mathrm{~V}$; Acquisition time $2 \mathrm{~min}$; Scan speed $2.1 \mathrm{scan} / \mathrm{s} ; \mathrm{m} / \mathrm{z}$ 100-3000 mass range. The NanoMate HCT MS system was tuned to operate in the positive ion mode. This technique was chosen, as protein and peptide ionization shows high ionization efficiency in the positive ion mode. The source block, maintained at the constant temperature of $80{ }^{\circ} \mathrm{C}$, provided an optimal desolvation of the generated droplets without a need of a desolvation gas. In all experiments, the desolvation gas was maintained at 30-50 liters per hour. For prevention of any cross contamination or carry-over, the pipette tip was ejected and replaced with a new one, after every sample infusion and MS analysis. All mass spectra were processed by the Data Analysis 3.4 software from Bruker Daltonik (Bremen Germany). Mass spectra were calibrated using sodium iodide as a calibrating agent. The accuracy on the determination of the average mass was $20 \mathrm{ppm}$, with a resolution of about 4000 . Samples were dissolved in methanol at a concentration of about $5 \mathrm{pmol} / \mu \mathrm{L}$. For an acquisition time of $2 \mathrm{~min}$, the required volume of sample was about 2 pmols, a value which reflects a very high sensitivity analysis.

\subsection{Study of the Biological Activity}

HeLa cells were grown in DMEM-Ham F12 medium (Sigma-Aldrich, Munich, Germany), supplemented with 10\% fetal bovine serum (Gibco, Basel, Switzerland) and 1\% antibiotic-antimycotic solution (Lonza, Cologne, Germany). They were seeded at a density of $10^{4}$ cells / well in Hi-Q ${ }^{4} 35 \mathrm{~mm}$ dishes (Ibidi GmbH, Martinsried, Germany) After cell attachment $(2 \mathrm{~h})$, the medium was replaced 
with a fresh volume supplemented with various concentrations of $S_{5}$ and $S_{2 T}$ lyophilized fractions $(1,2$ and $4 \mu \mathrm{g} / \mathrm{mL}$ respectively, dissolved in culture medium). For the control group, the medium was aspirated and replaced with fresh standard medium. The dishes were then placed inside the Biostation IM equipment (Nikon Corp., Kawasaki, Japan), a mini-incubator with an incorporated optical system and a CCD camera for timelapse imaging. The cells were monitored for $24 \mathrm{~h}$ and images were collected every $10 \mathrm{~min}$. To assess cell viability and proliferation, viable cells and mitoses were counted. For each experimental condition, images were collected from 5 different microscopic fields and the experiments were repeated three times. The wells which contained only the cells, without the added compounds, were considered as control.

\section{Conclusions}

This study was designed to investigate the chemical composition of extracts of a valuable medicinal plant, the hellebore, with therapeutic effects known since long ago in traditional medicine. The amino acids and peptides contained in a hellebore hydroalcoholic extract were identified and isolated. An appropriate isolation strategy was developed that can be applied for the isolation of such compounds from complex mixtures. The presented results indicate that the new isolation strategy and the feasibility of the developed characterization method show great potential as efficient separation methods for amino acids and peptides from natural products. The methods used in our study to isolate various fractions of the hellebore extract did not affect the antiproliferative activity, as shown by treating HeLa cells and monitoring their behavior by time lapse videomicroscopy.

Acknowledgments: This work was supported by Romanian National Authority for Scientific Research, through the Capacity-Module III: Bilateral cooperation programs: Project 628/2013 and Project 535/2012. A partial supplementary support was also obtained from the Romanian National Authority for Scientific Research, by the Project PN.09.33.02.05 and strategic grant POSDRU/159/1.5/S/134378 (2014) of the Ministry of National Education, Romania, co-financed by the European Social Fund-Investing in People, within the Sectoral Operational Programme Human Resources Development 2007-2013.

Author Contributions: Adina-Elena Segneanu, Ioan Grozescu: design of experiments, coordination of the lab work related to chemical investigations, results' interpretation and preparing the manuscript. Mircea Leabu: design of experiments, coordination of the lab work related to biological activity investigation, results' interpretation, and preparing the manuscript. Florentina Cziple, Daniel Berki and Daniel Damian: experiments for analitical investigations, Cristina Mariana Niculite, Alexandru Florea: experiments for biologic activity investigation, analysis of timelapse videomicroscopy results, typing the draft regarding the experiments they conduct, final reading of the manuscript for English accuracy. Florentina Cziple contributed with reagents/materials/analysis tools for chemical investigation; Daniel Berki and Daniel Damian typing the draft of manuscript.

Conflicts of Interest: The authors declare no conflict of interest.

\section{References}

1. Colegate, S.M.; Molyneux, R.J. Bioactive Natural Products Detection, Isolation and Structural Determination, 2nd ed.; Taylor \& Francis Group: Boca Raton, FL, USA, 2008; pp. 9-16, 249-261.

2. Ilie, T.; Minoiu, M. Plante Medicinale Miraculoase din Flora Romanie; Artmed: Bucuresti, Romania, 2004; pp. 225-227.

3. Domokos, E. The Ecological Characterization of the Forestry Associations from the Middle Stream of the Niraj Valley (Romania, Mureş County). Sci. Ann. Alexandru Ioan Cuza Univ. Iasi. 2013, 59, 99-106.

4. Neacsu, C.; Ciobanu, C.; Barbu, I.; Toader, O.; Szegli, G.; Kerek, F.; Babes, A. Substance MCS-18 Isolated from Helleborus purpurascens Is a Potent Antagonist of the Capsaicin Receptor, TRPV1, in Rat Cultured Sensory Neurons. Physiol. Res. 2010, 59, 289-298. [PubMed]

5. Kerek, F. Boicil, a new and very efficient antialgic spasmolytic and blood vessel regulating drug obtained from the plant Helleborus. Int. Conf. Chem. Biotechnol. Biol. Nat. Prod. 1981, 2, $22-37$.

6. Rosselli, S.; Maggio, A.; Bruno, M.; Spadaro, V.; Formisano, C.; Irace, C.; Maffettone, C.; Mascolo, N. Furostanol Saponins and Ecdysones with Cytotoxic Activity from Helleborus bocconei ssp. Intermedius. Phytother. Res. 2009, 23, 1243-1249. [CrossRef] [PubMed] 
7. Paun-Roman, G.; Neagu, E.; Radu, G.L. Membrane Processes for the Purification and Concentration of Helleborus purpurascens Extracts and Evaluation of Antioxidant Activity. Rev. Chim. Buchar. 2010, 61, 877-881.

8. Vochita, G.; Mihai, C.T.; Gherghel, D.; Iurea, D.; Roman, G.; Radu, G.L.; Rotinberg, P. New Potential Antitumoral Agents of Polyphenolic Nature Obtained from Helleborus purpurascens by Membranary Micro- and Ultrafiltration Techniques. Sci. Ann. Alexandru Ioan Cuza Univ. Iasi. 2011, XII, 41-51.

9. Stanescu, U.; Hanceanu, M.; Miron, A.; Aprostoaie, C. Plante Medicinale de la A la Z, Monografii ale Produselor de Interes Therapeutic; Grigore T. Popa University of Medicine and Pharmacy: Iasi, Romania, 2004; Volume 1, pp. 280-282.

10. Stroescu, V. Pharmacological Basis of Medical Practice; Medical Printing House: Bucuresti, Romania, 1998; pp. 236-241.

11. Simard, E.P.; Engels, E.A. Cancer as a Cause of Death among People with AIDS in the United States. Clin. Infect. Dis. 2010, 51, 957-962. [CrossRef] [PubMed]

12. Tantry, M.A. Plant natural products and drugs: A comprehensive study. Asian J. Tradit. Med. 2009, 4, 241-249.

13. Molinski, T.F.; Lievens, S.L.; Saludes, J.P. Drug development from marine natural products. Nat. Rev. Drug Discov. 2009, 8, 69-83. [CrossRef] [PubMed]

14. Wittmann, V. Glycoproteins: Occurrence and Significance, Glycoscience: Chemistry and Chemical Biology; Fraser-Reid, B.O., Tatsuta, K., Thiem, J., Eds.; Springer: Berlin/Heidelberg, Germany, 2008.

15. Caleya, R.F.D.; Hernández-Lucas, C.; Carbonero, P.; García-Olmedo, F. Gene expression in alloploid: Genetic control of lipopurothionins in wheat. Genetics 1976, 83, 687-699. [PubMed]

16. Caleya, R.F.D.; Gonzalez Pascual, B.; Garcia Olmedo, F.; Carbonero, R. Susceptibility of phytopathogenic bacteria to wheat purothionins in vitro. Appl. Microbiol. 1972, 23, 998-1000.

17. Ebrahimnesbat, F.; Behnke, S.; Kleinhofs, A.; Apel, K. Cultivar specific differences in the distribution of cell wall bound thionins during the generation of compatible and incompatible interactions between barley and powdery mildew. Planta 1989, 179, 203-210. [CrossRef] [PubMed]

18. Evans, J.; Wang, Y.; Shaw, K.P.; Vernon, L.P. Cellular responses to Pyrularia thionin are mediated by $\mathrm{Ca}^{2+}$ influx and phospholipase A2 activation and are inhibited by thionin tyrosine iodination. Proc. Natl. Acad. Sci. USA 1989, 86, 5849-5853. [CrossRef] [PubMed]

19. Bhave, M.; Methuku, D.R. Small Cysteine-Rich Proteins from Plants: A Rich Resource of Antimicrobial Agents, Science against Microbial Pathogens: Communicating Current Research and Technological Advances; Mendez-Vilas, A., Ed.; Formatex Research Center: Badajoz, Spain, 2011; pp. 1074-1083.

20. Apetrei, N.S.; Lupu, A.R.; Calugaru, A.; Kerek, F.; Szegli, G.; Cremer, L. The antioxidant effects of some progressively purified fractions from Helleborus purpurascens. Romanian Biotechnol. Lett. 2011, 16, 6673-6682.

21. Horstmann, B.; Zinser, E.; Turza, N.; Kerek, F.; Steinkasserer, A. MCS-18, a novel natural product isolated from Helleborus purpurascens inhibits dendritic cell activation and prevents autoimmunity as shown in vivo using the EAE model. Immunobiology 2008, 212, 839-853. [CrossRef] [PubMed]

22. Lupu, A.R.; Cremer, L.; Kerek, F.; Calugaru, A. New natural compound MCS-18. A TLR-2 antagonist able to down-regulate inflammation-related pain. Eur. J. Pain. 2009, 13, 527. [CrossRef]

23. Yang, F.Y.; Su, Y.F.; Wang, Y.; Chai, X.; Han, X.; Wu, Z.-H.; Gao, X.-M. Bufadienolides and phytoecdystones from the rhizomes of Helleborus thibetanus (Ranunculaceae). Biochem. Syst. Ecol. 2010, 38, 759-763. [CrossRef]

24. Neda, I.; Vlazan, P.; Pop, R.O.; Sfirloaga, P.; Grozescu, I.; Segneanu, A.E. Peptide and Amino Acids Separation and Identification from Natural Products. In Analytical Chemistry; Ira, S.K., Ed.; Intech: Rijeka, Croatia, 2012; pp. 135-146.

25. Sasidharan, S.; Chen, Y.; Saravanan, D.; Yoga Latha, L. Extraction, isolation and characterization of bioactive compounds from plants' extracts. Afr. J. Tradit. Complement. Altern. Med. 2011, 8, 1-10. [CrossRef] [PubMed]

26. Abdul Md, M.; Syed, M.T.; Apurba, S.; Debasish, B.; Mohammad, S.I. Isolation and Identification of compounds from the Leaf Extract of Dillenia indica Linn. Bangladesh Pharm. J. 2010, 13, 49-53.

27. Moughan, P.J. Amino acid availability: Aspects of chemical analysis and bioassay methodology. Nutr. Res. Rev. 2003, 16, 127-141. [CrossRef] [PubMed] 
28. Zamfir, A.D.; Serb, A.; Vukelic, Ž.; Flangea, C.; Schiopu, C.; Fabris, D.; Kalanj-Bognar, S.; Capitan, F.; $\mathrm{Sisu}, \mathrm{E}$. Assessment of the molecular expression and structure of gangliosides in brain metastasis of lung adenocarcinoma by an advanced approach based on fully automated chip-nanoelectrospray mass spectrometry. J. Am. Soc. Mass Spectrom. 2011, 22, 2145-2159. [CrossRef] [PubMed]

29. Kerek, F. Peptide Having a High Cysteine Content. U.S. Patent 7750114 B2, 6 July 2010.

30. Milbradt, A.G.; Kerek, F.; Moroder, L.; Renner, C. Structural characterization of hellethionins from Helleborus purpurascens. Biochemistry 2013, 42, 2404-2411. [CrossRef] [PubMed]

31. Niculite, C.; Leabu, M. Timelapse monitoring of cell behavior as a tool in tissue engineering. IFMBE Proc. 2011, 36, 352-357.

32. Rosu, T.; Pahontu, E.; Ilies, D.C.; Georgescu, R.; Mocanu, M.; Leabu, M.; Shova, S.; Gulea, A. Synthesis and characterization of some new complexes of $\mathrm{Cu}(\mathrm{II}), \mathrm{Ni}(\mathrm{II})$ and $\mathrm{V}(\mathrm{IV})$ with Schiff base derived from indole-3-carboxaldehyde. Biological activity on prokaryotes and eukaryotes. Eur. J. Med. Chem. 2012, 53C, 380-389. [CrossRef] [PubMed]

33. Riguera, R. Isolating bioactive compounds from marine organisms. J. Mar. Biotechnol. 1997, 5, 187-193.

34. Kaźmierczak, D.; Ciesielski, W.; Zakrzewski, R. Separation of Amino Acids as Phenyl Thiocarbamyl Derivatives by Normal and Reversed-Phase Thin-Layer Chromatography. J. Planar Chromatogr. 2005, 18, 427-431. [CrossRef]

35. Sanchez-Machado, D.I.; Lopez-Cervantes, J.; Lopez-Hernandez, J.; Paseiro-Losada, P.; Simal-Lozano, J. High Performance Liquid Chromatographic Analysis of Amino Acids in Edible Seaweeds after Derivatization with Phenyl Isothiocyanate. Chromatographia 2003, 58, 159-163.

36. Heinrikson, R.L.; Meridith, S.C. Amino acid analysis by reverse-phase high-performance liquid chromatography: Precolumn derivatization with phenylisothiocyanate. Anal. Biochem. 1984, 136, 65-74. [CrossRef]

37. Ebert, R.F. Amino acid analysis by HPLC: Optimized conditions for chromatography of phenylthiocarbamyl derivatives. Anal. Biochem. 1986, 154, 431-435. [CrossRef]

38. Cohen, S.A.; Strydom, D.J. Amino acid analysis utilizing phenyliosthiocyanate derivatives. Anal. Biochem. 1988, 174, 1-16. [CrossRef]

39. Dimova, N. RP-HPLC Analysis of Amino acids with UV-detection. C. R. Acad. Bulg. Sci. 2003, 56, 12-75.

Sample Availability: Samples of the compounds are not available from the authors.

(C) 2015 by the authors; licensee MDPI, Basel, Switzerland. This article is an open access article distributed under the terms and conditions of the Creative Commons by Attribution (CC-BY) license (http://creativecommons.org/licenses/by/4.0/). 